\title{
EXPLANATORY ASYMMETRIES: LAWS OF NATURE REHABILITATED
}

\author{
LINA JANSSON \\ PENULTIMATE DRAFT \\ FINAL DRAFT FORTHCOMING IN THE JOURNAL OF PHILOSOPHY
}

\section{Laws of Nature and Explanation: Some Preliminaries}

The intuitive pull towards accepting subsumption under natural laws as a form of explanation is very strong. However, the idea that subsumption under laws is explanatory famously fell on hard times with the onslaught of counterexamples to the deductive-nomological account; especially since that account spells out the intuition that laws can explain in a very natural and straightforward way by thinking of explanations as arguments that proceed (essentially) from laws. Among the counterexamples, the problem of explanatory non-symmetry is particularly troubling.

Whether or not one takes the relation of explanation to be by its nature asymmetric (and I do not), there are many cases to illustrate that explanation is not symmetric. Several such cases are by now familiar. For example, while it seems as if the height of a flagpole can play a role in explaining the length of the shadow it casts, it does not seem as if the length of the shadow can play this role in explaining the height of the flagpole. ${ }^{1}$ Examples of explanations that are not symmetrical are found throughout the sciences and are present both in cases that explicitly involve approximations and in instances of what could be taken to be foundational theories. For example, while the length of a simple pendulum might be taken to be part of an explanation of its period, the reverse does not hold. Similarly, while the acceleration experienced by one of two gravitating bodies can be explained by the existence of a distant mass, the mass of that distant body cannot similarly be explained by the acceleration.

Date: 23 July 2015.

I owe a great deal to many graduate students, faculty members, and visiting speakers at the University of Michigan for helpful discussions, comments, suggestions, and criticisms. Thank you also to the audiences at the Pitt-CMU twelfth annual graduate conference, Larryfest at Michigan, the NUS seminar series, the 2013 Swedish Congress of Philosophy, the HKU seminar series, and Explanation Beyond Causation at MCMP. Thank you to the anonymous referees for for very helpful suggestions. Many thanks to my colleagues at NTU. Part of this work was supported by a start-up grant from Nanyang Technological University.

${ }^{1}$ This case was put forward (with slightly different details) by Sylvain Bromberger. Why-Questions. In R. Colodny, editor, Mind and Cosmos, volume 3 of University of Pittsburg Series in the Philosophy of Science, pages 86-111. University of Pittsburg Press, 1966. 
These cases are troubling for the deductive-nomological account since the law that allows us to derive the length of the shadow from the height of the flagpole equally allows us to derive the height of the flagpole from the length of the shadow (and similarly for the other two cases). Although the failure of explanation to be symmetric is a problem directly only for the deductive-nomological account, it raises doubts about whether any account that allows non-causal dependencies, such as nomological dependencies, to play the central explanatory role is viable. Lipton nicely summarises the worry. In abandoning the deductive-nomological account all non-causal foundations for explanation, such as nomological ones, have come to be seen as suspect.

The existence of non-causal explanations show that a causal model of explanation cannot be complete. One reaction would be to attempt to expand the notion of causation to some broader notion of 'determination' that would encompass the noncausal cases (Ruben 1990: 230-3). This approach has merit, but it will be difficult to come up with such a notion that we understand even as well as causation, without falling into the relation of deductive determination, which will expose the model to many of the objections to the deductive-nomological model. ${ }^{2}$

The ability to solve the problem of explanatory non-symmetry in a simple and elegant way is one of the main attractions of a causal account of explanation. Here laws can do explanatory work, but they are no longer independently explanatory. Rather, they can do explanatory work in so far as they are causal laws. ${ }^{3}$ For example, Strevens's account tries to remain as metaphysically neutral as possible, but when it comes to how the account handles explanatory non-symmetry this neutrality runs out.

All religions, however ecumenical, need someone to burn. And there is, not surprisingly, a view of the nature of causation that is ruled out by even my ecumenism. I have in mind an emergent view of causation, on which there are no causal relations at the level of fundamental physics ... To abandon this possibility is no loss for the proponent of the causal approach to explanation, however, since it is the one view of causation that clearly cannot solve the problem of explanatory asymmetry.

\footnotetext{
${ }^{2}$ Peter Lipton. Inference to the Best Explanation, second edition. Routledge, 1991/2004, p 32.

${ }^{3}$ This line of reasoning is prominent in several recent sophisticated accounts of causal explanation such as those of James Woodward. Making Things Happen: A Theory of Causal Explanation. Oxford University Press, 2003, chapter 6 and Michael Strevens. Depth: An Account Of Scientific Explanation. Harvard University Press, 2008 , chapter 7.
} 
... [W] hat I assume in the way of causal relations is ... just what is needed, and no more, to provide a causal resolution of the explanatory asymmetries. ${ }^{4}$

This solution is extremely elegant. However, there are also many examples of explanations that are, at least plausibly, non-causal. ${ }^{5}$ This may not seem to be a problem for causal accounts that are willing to be pluralist about explanation and to claim to have accounted only for causal explanation. ${ }^{6}$ I have argued elsewhere that pluralism does not come cheaply. ${ }^{7}$ Roughly and in brief, the difficulty is that causal accounts solve the problem of explanatory non-symmetry by demanding that only causal information is explanatory (at least within a non-ad hoc delineated domain such as all explanation of physical events). Without this exclusivity we no longer have a solution to the problem that certain law-based derivations are non-explanatory. All that we would be allowed to conclude is that such law-based derivations are not causal explanations. Without assuming causal explanatory exclusivity they could be explanations nonetheless. An explanatory pluralist who rejects causal explanatory exclusivity needs a non-causal solution to the problem of explanatory non-symmetry for these cases.

I will argue that we have the resources to solve the problem of non-symmetry without invoking causal notions. This will not rescue the deductive-nomological account. However, it will rehabilitate one of the central ideas from that account by showing that laws of nature can provide the basis for explanation without running afoul of the problem of explanatory non-symmetry.

The challenge of accounting for particular explanatory asymmetries is only one of several difficulties that faced the deductive-nomological account. Yet, I take the problem of explanatory non-symmetry to be the most serious challenge to the general idea that laws of nature can do independent explanatory work and to be the problem that most strongly favours causal account of explanation. In order to address counterexamples put forward to the deductive aspect of the

\footnotetext{
${ }^{4}$ Michael Strevens. Depth: An Account Of Scientific Explanation. Harvard University Press, 2008, pp. 34-35. ${ }^{5}$ For some recent discussions see for example Collin Rice. Moving Beyond Causes: Optimality Models and Scientific Explanation. Nô̂s, DOI: 10.1111/nous.12042, forthcoming; Marc Lange. What Makes a Scientific Explanation Distinctively Mathematical? British Journal for the Philosophy of Science, 64(3):485-511, 2013, and Alisa Bokulich. Reexamining the Quantum-Classical Relation: Beyond Reductionism and Pluralism. Cambridge University Press, 2008. For a discussion of many cases and a defence of the claim that all explanations of particular events are causal see Bradford Skow. Are There Non-Causal Explanations (of Particular Events)? British Journal for the Philosophy of Science, 65(3):445-467, 2014.

${ }^{6}$ I take this to be the view of Woodward and Strevens.

${ }^{7}$ See Lina Jansson. Causal Theories of Explanation and the Challenge of Explanatory Disagreement. Philosophy of Science, 81(3):332-348, 2014.
} 
deductive-nomological account it is possible to appeal to what Woodward ${ }^{8}$ calls "the hidden structure strategy". ${ }^{9}$ For many of the other counterexamples one might hope for a solution by simply being more careful about what counts as a law, and, in particular, by being careful to note any implicit ceteris paribus clauses and/or conditions of application. ${ }^{10}$ However, no matter how successful these strategies turn out to be, on their own these strategies do not help with the problem of explanatory non-symmetry. After all, there will still be cases where the law is bona fide, ceteri are pares, and the conditions favour applicability so that the derivation can go ahead (or else the account would leave us with no explanations at all), but if this is the case for the derivation that is explanatory then it will be the case for the derivation that is non-explanatory too. Without a solution to the problem of explanatory non-symmetry law-based accounts cannot even get off the ground.

Before going further, let me pause briefly to clarify the kind of account that I will go on to present and that I take the traditional models to be. First, the concern is mainly with explaining why events, regularities, etc., occur or why they have certain features (as opposed to offering explanations as mere reasoning heuristics, etc.). Second, within accounts of explanation there is a descriptive project of trying to give conditions that capture our explanatory practices, as they actually are, and also a normative project of trying to provide conditions for what our explanatory practices ought to (perhaps ideally) be like. I take the standard models to give prescriptive accounts, and that is what I will be doing too. This does not mean that the descriptive project is irrelevant to these accounts or to mine. I take them and myself to be involved in a project of spelling out what it is that we aim towards in our explanatory practices. Rather than being understood as imposing norms external to the practice the project is better seen as an explication of the normative commitments that we already employ (although perhaps not explicitly) together with the assumption that our practices are not thoroughly and radically misguided (at least in central and well-understood cases).

\footnotetext{
${ }^{8}$ James Woodward. Scientific Explanation. In E. Zalta, editor, The Stanford Encylopedia of Philosophy. http://plato.stanford.edu/archives/win2014/entries/scientific-explanation/, 2003/2014.

${ }^{9}$ See for example Peter Railton. Probability, explanation, and information. Synthese, 48(2):233-256, 1981 for the use of ideal texts or Philip Kitcher. Explanatory Unification and the Causal Structure of the World. In P. Kitcher and W. Salmon, editors, Scientific Explanation, volume XIII of Minnesota Studies in the Philosophy of Science, pages 410-505. University of Minnesota Press, 1989, section 5, for a defence of deductive chauvinism.

${ }^{10}$ In particular, one might try to avoid many of the problems of causal pre-emption, common cause and at least some of the cases of explanatory irrelevancies in this way. David-Hillel Ruben. Explaining Explanation. Routledge, 1990 considers, but rejects, this strategy in chapter VI. However, Ruben's discussion is operating under the assumption that the notion of law should respect logical empiricist sensibilities.
} 
Moreover, I will primarily be focused on canonical deterministic explanation. I take there to be many and important norms of explanation that have to do with idealisations, simplifications, etc., that I will not address here. I will not argue for the merit of such an approach independently; here the proof of the pudding is in the eating. I will simply display the virtues of the approach by showing that there are fairly intuitive criteria that we impose on such explanations and that understanding these enable us to tackle the problem of non-symmetry for many non-canonical explanations too. Finally, since I do not think that our judgements of explanatory asymmetry in the specific cases commonly invoked in the literature merit us drawing the conclusion that explanation is always asymmetrical, I take it to be enough to successfully address the problem of explanatory non-symmetry if I can show how to account for the judgements of asymmetry in these cases.

\section{What LaWs Are versus What Laws Do}

The first challenge encountered when trying to address the role of laws in explanation is the wide range of views about what laws of nature are. I will not rely on any particular such theory. I will instead be focused on what laws do. That is, I will be concerned with the role that they play within our theories. ${ }^{11}$ This strategy raises an immediate worry. Since I will remain silent on what the laws of nature metaphysically are, it is possible that our best such account will end up making use of the notion of explanation. I think that we have some defeasible reason to think that this is not the case. Judgements that separate laws from accidents do not seem to require us to make explanatory judgements. After all, it is not the case that any event, property, state, etc., in a nomological relationship can explain any other that it is so related to (as we have seen in the asymmetry counterexamples facing the deductive-nomological account). Finally, even if our best account of laws ultimately does have to rely on judgements about explanatoriness, the account offered of explanation in this paper will still be an informative (although not reductive) one. In many debates over explanation the status of the putative law as a law is not in question. ${ }^{12}$

The assumption about the role of laws within a theory that the rest of this paper will rely on is this: laws allow us to make inferences beyond those of deductive logic. I take it that the laws of a given theory are of particular interest at least partially because they give us information about

\footnotetext{
${ }^{11}$ John Roberts. The Law-Governed Universe. Oxford University Press, 2008 builds an account of laws around one conception of the role that laws play in our theories, but this will not be required for the account that I give here.

${ }^{12}$ Moreover, similar assumptions are in play in causal accounts of explanation.
} 
the kind of inferential moves that the theory sanctions. This is reminiscent of the notion of laws of nature as "inference tickets" that Ryle ${ }^{13}$ mentions. However, I will only rely on this being one of the roles that laws play. I make no claim as to the primacy of this role.

With this in place, let me now go on to consider some restrictions that our theories and practice typically impose on the inferential use that we can make of what is stated as laws within the theory.

2.1. The role of conditions of application. Take as an example a version of the second law of Newtonian mechanics, namely, the claim that force, $\vec{F}$, is given by the product of mass and acceleration, $m \vec{a}$. Although the law is often presented in this form, the introduction to the law will come with significant clarifications. First, this law only allows us to move from finding out that the net force on some body, $m$, has some magnitude and direction to the claim that $m \vec{a}$ has the same magnitude and direction. ${ }^{14}$ In thinking of this law as licensing certain inferences we can imagine that there is a rule, relative to Newtonian mechanics, that tells us that we are allowed to move from $\vec{F}_{\text {net }}$ to $m \vec{a}$ (and sometimes vice versa).

Similarly, when spelling out the inferences that are licensed by the law of gravity we have to take some care in how we understand $\vec{F}=-\frac{G M m}{|r|^{2}} \hat{r} .{ }^{15}$ We can, for example, specify that we are dealing with only the component of the force from the gravitational interaction of point-like masses $m$ and $M$ (the option that I will take here). In this case we should think of the law as $\vec{F}_{\text {gravity }(\text { from the } m-M \text { interaction })}=-\frac{G M m}{|r|^{2}} \hat{r}^{16}$

In order to use the two laws above to derive the claim that $\vec{a}=-\frac{G M}{|r|^{2}} \hat{r}$, we need some additional assumptions. In particular, we need to assume that we have two massive gravitating bodies where the force from the gravitational interaction is the only net force acting on the bodies and that these bodies can be treated as point-like masses. In general it will simply be false that, for example, when the acceleration of some mass $m$ is non-existent (set to 0 ) then the value of the other mass, $M$, is set to 0 too.

When we try to understand the role that the laws are playing, it is crucial to look to the broader theory and the conditions of application. It would be a mistake to think that we had shown that

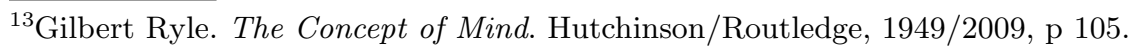

${ }^{14}$ We would have to be even more careful if we do not assume that it is the net force on some body with constant mass that we are dealing with.

${ }^{15} \mathrm{I}$ am using the convention that $\hat{r}$ is a unit vector.

${ }^{16} \mathrm{My}$ approach here is very much in line with that of Carl Hempel. Provisoes: A problem concerning the inferential function of scientific theories. Erkenntnis, 28(2):147-164, 1988, p 151.
} 
$\vec{a}=-\frac{G M}{|r|^{2}} \hat{r}$ was false by looking at a system that is subject to a force contributing to the net force that is not gravitational. Rather, $\vec{a}=-\frac{G M}{|r|^{2}} \hat{r}$ simply does not apply to such a system. ${ }^{17}$ Moreover, as this example illustrates, what would allow us to combine two laws in a derivation might demand assumptions that are different from the ones (if any) that are required in understanding the role that each typically plays (here one such additional assumption is that the only net force is the force from the gravitational interaction). ${ }^{18}$

On its own this is not a novel claim. Cartwright in particular has long argued for the crucial importance of such considerations (although in more general terms than I accept). Moreover, she sees them as raising a problem for law-based explanations.

For most cases, either we have a law that purports to cover, but cannot explain because it is acknowledged to be false, or we have a law that does not cover. Either way, it is bad for the covering-law picture. ${ }^{19}$

Unlike Cartwright, I do not think that the desiderata of explanation and application are in conflict. My argument throughout the rest of this article will attempt to show that the conditions of application are part of our explanatory practices, and they will play a crucial role in allowing explanations based on laws to evade the non-symmetry problem.

\section{Flagpoles and their shadows, masses and aCCelerations, the lengths AND PERIODS OF SIMPLE PENDULA}

With the warning to pay attention to conditions of application in place, we can now move on to look at some of the central examples of explanatory asymmetry in the literature. First, let us revisit the well-worn case of the flagpole and the shadow.

3.1. Flagpoles and shadows. Let $y$ be a variable for the height of the flagpole and $H$ be the height of the specific flagpole in question. Similarly, let $x$ be the variable for the length of the shadow and $L$ the length of the specific shadow. Finally, let $z$ be a variable for the angle the sun makes with the horizon at the location of the flagpole and the shadow, and let $a$ be a specific such

\footnotetext{
${ }^{17}$ For reasons that John Earman and John Roberts. "Ceteris Paribus", There Is No Problem of Provisos. Synthese, 118(3):439-478, 1999 present, I do not think that all laws of physics are ceteris paribus ones. This is why I am treating these as conditions of application rather than as ceteris paribus clauses.

${ }^{18}$ Notice that even if we take the law of gravity and the second law to apply unrestrictedly this point still stands for the derived law.

${ }^{19}$ Nancy Cartwright. How the Laws of Physics Lie. Clarendon Press, Oxford, 1983/2002, p 45-46.
} 
angle. Let us also assume that none of $H, L$ and $a$ are zero and that $H=L \tan a$.

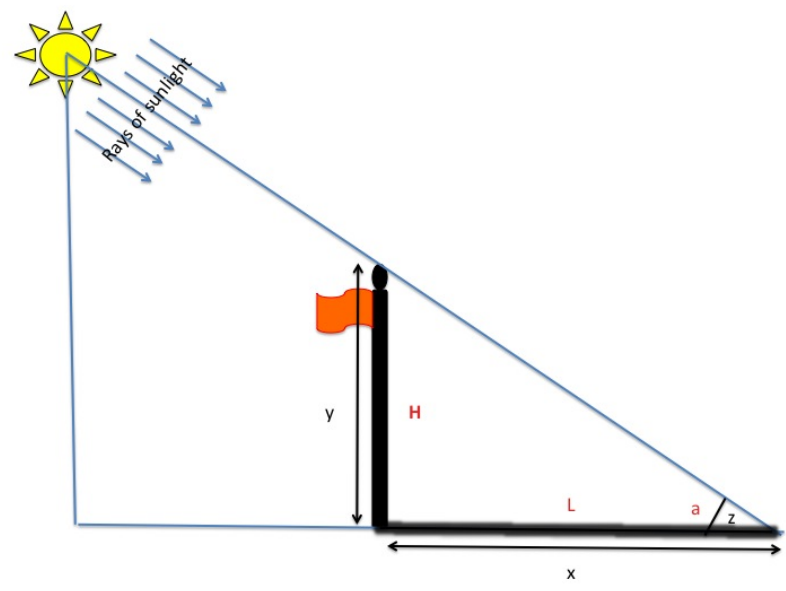

Figure 1. Flagpole and its shadow

putative explanation 1.

Relation: $y=x \tan z$

Particular fact: $x=L$

Particular fact: $z=a$

Explanandum: $y=H$ putative explanation 2 .

Relation: $x=y / \tan z$

Particular fact: $y=H$

Particular fact: $z=a$

Explanandum: $x=L$

In putative explanation 1 the height of the flagpole is derived from the length of the shadow but is not explained by the length of the shadow, while, so the story goes, in putative explanation 2 the length of the shadow is both derived and explained by the height of the flagpole.

The relation that is playing the role of a law here is not straightforwardly recognisable as a law of any particular theory. What may be taken to be a law is that light moves in straight lines and from this, together with other assumptions, we can apply the general trigonometric relationship. We also know that when it comes to applying this relationship to flagpoles and their shadows there are a number of conditions that we impose in order to take the application to be legitimate. Most obviously, we know that the relationship will be misleading when there is no source of light (say because of a solar eclipse or because the sun is below the horizon). ${ }^{20}$ After all, even if the length of the shadow is 0 in these cases, we know that the height of the flagpole is not. This is not a problem

\footnotetext{
$\overline{20}$ There are several other assumptions needed, but focusing on this one will be sufficient to make the point clear.
} 
that we typically run up against when we try to apply the trigonometric relationship to flagpoles and shadows because we have a rough sense of when this relation is applicable to real flagpoles and their shadows and when it is not.

Of course, there will be cases where this condition is fulfilled and the derivation both in putative explanation 1 and putative explanation 2 can go ahead. This is what gives rise to the problem for the deductive-nomological account. However, the condition of application gives rise to a difference in the patterns of counterfactual sensitivity in the two cases. When the condition holds, we know how the length of the shadow varies with the height of the flagpole. Moreover, we also know something about how the actual value of the length of the shadow (in this case a non-zero value) would vary when this condition of applicability is not fulfilled. In this case, when the assumption that there is a light source fails then there will not be a shadow. However, this is not the case for the height of the flagpole. The height of the flagpole is not sensitive to violations of the condition of applicability. Even when the assumption that there is a light source is violated the height of the flagpole would not vary.

So far, I have not relied on any information that has to (even though it can) be understood causally. After all, simple observation (and generalisation of observation) allows us to conclude that flagpoles do not change their height, for example, at night. While the conditions of application are ones that the length of the shadow seems to be (counterfactually) sensitive to, this is not the case for the height of the flagpole. This will provide the foundation for my diagnosis of these cases in the next section.

However, before moving on to consider the general case, let me briefly note how this applies to other common cases of explanatory asymmetry and show that it is not a feature peculiar to the case of flagpoles and shadows.

3.2. Masses and accelerations. Asymmetries when it comes to specific explanatory relations are pervasive. I have already noted that if we have a system with two gravitating bodies then we take the presence of a distant body to explain the acceleration that the other body experiences. However, we do not take this acceleration to explain the mass of the distant body. Here too there is a difference that stems from the counterfactual sensitivity to the conditions of applicability. In section $2.1 \mathrm{I}$ argued that in order to make the reasoning in either direction legitimate we need to assume that there are no forces other than the one from the from the gravitational interaction 
acting on the bodies. ${ }^{21}$ If there was another force acting on the mass in question, we know that the acceleration of this mass would be sensitive to this and it would, typically, be different from its supposed actual value. However, this is not the case for the reverse direction. The mass of the distant body is not sensitive to the existence of another net force acting on the other body. Here too we have a difference in sensitivity to violations of the assumptions required for the (in this case derived) law to apply.

3.3. Lengths and periods of simple pendula. We find this pattern repeated yet again when it comes to the conditions required in order to make use of the simplified (and approximate) equation governing the behaviour of a simple pendulum. Here (like in the flagpole case) we are making many assumptions, and it will be enough for my purposes to focus on a few of them. The simplified behaviour is described by $T \approx 2 \pi \sqrt{\frac{L}{g}}$, where $T$ is the period, $L$ is the length and $g$ is the local acceleration due to gravity. The first feature of this equation to stress is that it is an approximation for small swings and that it only holds in the absence of other forces such as air resistance. Here too we find a difference in the sensitivity to the conditions of application between the period $T$ and the length $L$. Neither the presence of air resistance nor large swings would indicate a different value for the length of the pendulum. The length, unlike the period, does not generally seem sensitive to violations of the conditions of application that the law comes with.

\section{LAW-BASED DEPENDENCE}

The difference in the counterfactual sensitivity on the conditions of applicability that I have noted in the cases above points us in the direction of a systematic solution. In the rest of this section I will show how sensitivity to the applicability criteria for the laws matters for explanatory purposes.

The basic intuition that I will make use of is very simple. The difference between mere description of a phenomenon and an explanation of that same phenomenon lies in whether information about what the phenomenon depends on has been provided. For example, the length of the shadow does not explain the height of the flagpole since the height of the flagpole does not depend on the length of the shadow. However, the height of the flagpole can explain the length of the shadow since the length of the shadow does depend on the height of the flagpole. Similarly, if the stability of the

${ }^{21}$ Or if we treat the derived law as an approximation then we should demand that there are no other non-negligible forces. 
planetary orbits depends on the dimensionality of space-time then the stability of the planetary orbits is (at least in part) explained by the dimensionality of space-time. ${ }^{22}$

Hopefully this sounds rather commonsensical, so it is worth noting how this driving intuition differs from that of some other accounts of explanation. In particular, this motivation differs from the one in the deductive-nomological account where to explain is to show why the phenomenon was to be expected given the laws of nature. It is also distinct from the motivation for unificationist accounts, such as those of Friedman ${ }^{23}$ and Kitcher ${ }^{24}$. Here to explain is to reduce the number of types of phenomena that have to be taken to be brute. Finally, although it is similar to the motivating intuition behind causal accounts of explanation, the dependence employed is broader than causal dependency (as the example of planetary orbits and the dimensionality of spacetime indicates). ${ }^{25}$

At this point it would be tempting to take the relevant notion of dependence to simply be counterfactual dependence. I was clearly invoking counterfactual scenarios in the examples throughout section 3. This is, of course, right, but the dependence is not simple counterfactual dependence. Counterfactual dependence is neither necessary nor sufficient for explanatory dependence. When we consider what it is that we are trying to capture, this is not surprising. When we explain the event, state, or property of an event, etc. $b$ by the event, state or property of an event, etc. $a$ by a law, we are interested in what this law tells us about the dependence of $b$ on $a{ }^{26}$ The notion of dependence is a modal one, so information about what would have happened in other circumstances

\footnotetext{
${ }^{22}$ This example is noted by James Woodward. Making Things Happen: A Theory of Causal Explanation. Oxford University Press, 2003, p 220-221 and is discussed by Paul Ehrenfest. In what way does it become manifest in the fundamental laws of physics that space has three dimensions? Proceedings of the Royal Netherlands Academy of Arts and Sciences, Amsterdam, 20(1):200-209, 1918; John D. Barrow. Dimensionality. Physical Transactions of the Royal Society of London. Series A, Mathematical and Physical Sciences, 310(1512):337-346, 1983 and Craig Callender. Answers in search of a question: "proofs" of the tri-dimensionality of space. Studies in History and Philosophy of Modern Physics, 36(1):113-136, 2005. If the dimensionality of space-time does not depend on the stability of the planetary orbits (which seems plausible) then the stability of the planetary orbits does not explain the dimensionality of space-time.

${ }^{23}$ Michael Friedman. Explanation and Scientific Understanding. The Journal of Philosophy, 71(1):5-19, 1974.

${ }^{24}$ Philip Kitcher. Explanatory Unification. Philosophy of Science, 48(4):507-531, 1981 and Philip Kitcher. Explanatory Unification and the Causal Structure of the World. In P. Kitcher and W. Salmon, editors, Scientific Explanation, volume XIII of Minnesota Studies in the Philosophy of Science, pages 410-505. University of Minnesota Press, 1989.

${ }^{25}$ There are other such notions around. For example, Jaegwon Kim. Explanatory Knowledge and Metaphysical Dependence. Philosophical Issues, 5(Truth and Rationality):51-69, 1994 includes mereological dependence in addition to causal dependence and takes the relevant notion to be asymmetrical and transitive. However, as I will argue below, I do not think that the kind of dependence Kim discusses will do the work required here. David-Hillel Ruben. Explaining Explanation. Routledge, 1990, p 230-233, also suggests an expansion from causal relations in terms of the relations that can do explanatory work, but Ruben does not go on to develop this account in detail.

${ }^{26} \mathrm{I}$ am leaving it open for now what the proper relata of laws should be.
} 
(and so some counterfactuals) is relevant. However, the straightforward counterfactual about what would have happened had a not obtained is not. An example that is modified (in order to express a counterfactual) but that otherwise parallels Sanford's ${ }^{27}$ discussion about dependence and conditionals illustrates this.

Example: If Jim had been fired then Bill would have been fired too.

Many different circumstances could be imagined that would serve to make the counterfactual statement true, but only some of them involve Bill's job depending on Jim's. Continuing the adaption of Sanford's case and discussion, it could be that Jim is the son of the employer and only if the company went bankrupt, bringing with it the firing of all employees (including Bill), would Jim be fired. ${ }^{28}$ Or perhaps Bill is a terrible worker, known to be disliked by his employer, and if anyone at all were to be fired then he would be sure to be the first one to go. ${ }^{29}$ In neither of these cases does Bill's firing depend on Jim's. In the first scenario they have a common cause: the insolvency of the company. In the second case they do not even have to have a common cause. The truth of the counterfactual is guaranteed by our knowledge that, whatever the cause of Jim's being fired, the employer would rather have fired Bill, so if Jim was fired then either Bill had already been let go for incompetence or he was fired for the same reason that Jim was. However, if Bill's job is contingent on Jim's job, perhaps because Bill is Jim's personal assistant, then Bill losing his job does depend on Jim losing his. ${ }^{30}$ As Sanford notes, these differences are not visible in the counterfactual statement above. For necessity, we have examples of preemption where causal and explanatory relations seem to hold, but the corresponding counterfactual does not.

Rather, the dependence required in the case of law-based explanation is a special kind of counterfactual dependence where we ask, roughly, whether the law in question is such that it guarantees that $b$ holds when $a$ does and that $b$ does not when $a$ does not. ${ }^{31}$ The requirement that it is the law that guarantees the relevant counterfactuals rules out cases where the law is simply an idle

$\overline{27}$ David Sanford. If P, then Q: Conditionals and the Foundations of Reasoning. Routledge, 1989/2003, p 192 forward. Sanford's cases do not concern counterfactuals, but they can easily be adapted to do so (as Sanford notes). It is possible to make the same point more quickly, but less clearly, by noting that D. Lewis. Causation. The Journal of Philosophy, 70(17):556-567, 1973 has to rule out backtracking counterfactuals.

${ }^{28}$ David Sanford. If P, then Q: Conditionals and the Foundations of Reasoning. Routledge, 1989/2003, p 193, case 4. ${ }^{29}$ David Sanford. If P, then Q: Conditionals and the Foundations of Reasoning. Routledge, 1989/2003, p 193, case 5. ${ }^{30}$ David Sanford. If P, then Q: Conditionals and the Foundations of Reasoning. Routledge, 1989/2003, p 193, case 1.

${ }^{31}$ Note that even if we take the view of Marc Lange. Laws \& Lawmakers: Science, Metaphysics, and the Laws of Nature. Oxford University Press, 2009 that subjunctive facts are foundational, we do not need to deny that we can ask questions about and evaluate such counterfactual scenarios. We do, however, have to be careful not to read the "guarantee" as a statement of metaphysical fundamentality. 
tag-along. ${ }^{32}$ For counterfactual dependence typically the only non-actual world relevant to the evaluation of whether $b$ depends on $a$ is the closest (or set of closest) possible not- $a$ worlds. For nomological dependence all and only those worlds where the law guaranteeing dependence holds are relevant to the evaluation. In principle these sets of worlds need not have any common members at all (although we expect that they often will since we expect that the closest possible not- $a$ world will typically be one where the actual laws of nature hold).

Before we can put the notion of nomological dependence to use in an account of explanation there is a final complication and extension of the use that I need to address. That is why the first statement was a rough one. So far I have only talked of there being one law guaranteeing the dependence of one event (or property, state, etc.) on another one. In general we expect there to be a collection of laws that together guarantee that one event (or property, state, etc.) depends on a collection of other events (properties, states, etc.). So far the extension is straightforward. However, as soon as we are dealing with dependence on a collection of phenomena there are two different ways in which this could happen. It could be that the laws guarantee dependence between the individual events (or properties, states, etc.) and the phenomenon to be explained. However, the laws can also do so between the phenomenon to be explained and a general condition instantiated by the various particular events, properties, states, etc., involved. In the second case the phenomenon to be explained directly depends on the general condition and indirectly on the component events (etc.) that instantiate it.

The second case is typical for nomological dependence. Laws typically do not relate individual events (etc.) directly. Instead, when we speak of dependence on a particular event, or a trope, or the object that instantiates the property, etc., the dependence is indirect. For example, when we speak of the final velocity of a ball with a certain mass depending on being hit by another ball with a certain mass and velocity in a certain kind of collision, etc., we do so while knowing that the law in question does not relate that specific ball to any other specific ball. Rather, the final velocity depends on there being an impact of a certain general kind. We take the velocity to depend on the specific balls and their specific properties since they instantiate the general condition. So, for example, we might say that the velocity after the collision depends on the mass and the initial

\footnotetext{
32 This formulation is deliberately different from the requirement in Carl Hempel and Paul Oppenheim. Studies in the Logic of Explanation. Philosophy of Science, 15(2):135-175, 1948 that the law plays an essential role in the derivation. The difference will not be very important in this paper, but it is important in general and in particular when dealing with necessary existents (such as those found in mathematics).
} 
velocity of the two balls even though the law only gives us a general relation between masses, initial velocities, and final ones in elastic collisions.

Finally, notice that this breaks the symmetry of the earlier, rough, formulation of nomological dependence. When we are dealing with the explanation of particular events, states, etc., it will in general be the case that we are explaining one event (state, etc.) by a collection of events (states, etc.) that instantiate a general condition that the target event (etc.) depends on. From this explanation we cannot infer that the dependence holds in the reverse direction. After all, the particular event (state, etc.) depends directly on a general condition and indirectly on the particular events (states, etc.) that instantiate it. This general condition is not itself an event or state (at least assuming some mild constraints on what is allowed to count as such). ${ }^{33}$

With this notion of dependence in place, the time has now come for explanation. The starting point is straightforward; the notion of dependence that I relied on in the intuitive motivating claim that to explain a phenomenon is to show what it depends on, is, in the cases of section 3 , nomological dependence. Spelling out this motivating intuition a little more carefully - with the caveat that I will only be concerned with deterministic, full and canonical explanation here-we can say that a phenomenon is explained by some phenomena and some laws between phenomena when those laws guarantee the dependence of the target phenomenon on the phenomena. To give a law-based explanation is then to describe those laws and show how they ensure the dependence of the target phenomenon on the phenomena doing the explaining. Even with the caveat in place this notion of nomological explanation is interesting since it gives us a way of understanding what it is that we aim towards when we give law-based explanations. This will give us a stronger notion than one that we typically actually fulfil, but I will argue that it nonetheless give us a way to understand the asymmetry of the examples in section 3 . In particular, it will allow us to understand the importance of whether or not the target phenomenon to be explained is sensitive to the conditions of application of the laws in question.

We are now finally in a position to give a more systematic diagnosis of the cases in section 3. If we assume that we are aiming to give information about a collection of laws that together guarantee the dependence of what is to be explained on what is doing the explaining then we have good reasons to think that some, and only some, instances of derivation from the laws in section 3

\footnotetext{
$\overline{33}$ I illustrate this in more detail on page 15.
} 
give us partial information about this collection of other laws and the dependence guaranteed by them.

For example, we have good reason to think that the application of the trigonometric relation in the flagpole and shadow case is not giving us partial information about a collection of laws that guarantees that the height of the flagpole depends on the length of the shadow. We have good reason to believe this since we know something about what the conditions of applicability of the trigonometric relationship are, and we have good reason to think that the height is not sensitive to a failure of these conditions of applicability.

What we would, ideally, like is something that fits the sketch below.

Explanandum: The height of the flagpole $y$ is $H$.

Particulars: The angle $z$ is $a$ and the length of the shadow $x$ is $L$.

General condition: The length of the shadow $x$ and the angle $z$ instantiate the general condition $x \tan z=H$.

Laws: There are laws $L_{1} \ldots L_{n}$ such that they

(1) license the inference from the general condition obtaining to the explanandum obtaining (and a spelling out of how they do so).

(2) license the inference from a failure of the general condition to obtain to the failure of the explanandum to obtain (and a spelling out of how they do so).

We have good reason to think that we do not only lack the ability to spell this sketch out in detail but that there is no way to spell it out. The trigonometric law does not describe the relationship between flagpoles and shadows without restrictions. One of the conditions of application of the trigonometric law is that there has to be a light source present, and when this is violated we find that the height of the flagpole is unchanged. This gives us good (but not infallible) evidence to support the conclusion that there is no collection of laws $L_{1} \ldots L_{n}$ available to fill out the sketch above. After all, this seeming lack of sensitivity to the condition of applicability indicates that we have not merely been unable to specify in more detail the laws that govern the dependence of the height of the flagpole on the presence or absence of light but that the presence or absence of light (and of a shadow) are not relevant factors at all.

In the case of the period and the length of the pendulum we know that there are situations where what we call the law (for the simple pendulum) has not been violated, but simply does not 
apply, and we know that in these situations the length would be at its actual value even when the period is not. This gives us good reason to hold that the law in question does not provide us with information about some part of a collection of laws that together ensure that the length depends on the period. After all, we know of situations where this appears to be false. ${ }^{34}$ Similarly, we have good reason to hold that the derived law of the acceleration experienced by a body does not provide us with information about some part of a collection of laws that ensure that the mass depends on the acceleration. After all, here too we know that this appears to be false when the conditions of application are not fulfilled.

However, things are different when it comes to the length of the shadow, the acceleration of the body, and the period of the pendulum. Without knowing more about the specific counterfactual scenario, we cannot in all of these cases specify exactly what will happen when the conditions of application of the law fails; however, we do know that their values will, in general, differ from the assumed actual values. For example, when there is no light source then there will be no shadow, when there is a different net force then the body will move differently, when there are dissipative forces present then the period will not be independent of time, etc. Here we can take ourselves to have partial information about a collection of laws that guarantee that the length, the acceleration and the period will be sensitive to variations in the height, the mass, and the length respectively. ${ }^{35}$

Moreover, none of these observations require us to reason causally. Although we can, of course, do so, simple observation is enough for us to know that when there is no light source then there is no shadow and that the length of the pendulum is not affected by stopping the swing of the pendulum, etc. In the case of Newton's laws of motion and the universal law of gravity we get the relevant conditions of application for the derived law from the theoretical understanding of the more basic laws. Here we can also have empirical support for the laws although it is of a much more sophisticated kind. ${ }^{36}$

\footnotetext{
${ }^{34}$ Of course there is a possibility of being deceived about this if we are unlucky enough to live in a systematically deceptive world. This, however, is not a feature peculiar to law-based explanation. We can similarly be deceived about the causal structure of a situation.

${ }^{35} \mathrm{I}$ am simplifying somewhat here by using the term "sensitive". We know that, for example, the height of the flagpole does not explain the length of the shadow on its own. Rather, it is part of the instantiation of a general condition that explains the length of the shadow. So the laws really guarantee that the length of the shadow depends on this condition directly and only indirectly on the actual height (and similarly for the other two cases).

${ }^{36}$ For a recent detailed discussion of how we can come to have such evidence see for example William Harper. Isaac Newton's Scientific Method: Turning Data into Evidence about Gravity and Cosmology. Oxford University Press, 2012.
} 
Now we are in a position to revisit how the view presented here relates to the traditional accounts of explanation. As I stressed earlier, the motivation is closest to the motivation behind causal accounts (such as Woodward's) in its focus on the notion of dependence. However, unlike causal accounts it is focused on nomological dependence and does not tackle failures of explanatory symmetry by appeal to causal asymmetry. Finally, in this last respect, this accounts invokes similar considerations to those developed by the unificationist account. Kitcher also considers the absence of light, etc., in accounting for the failure of symmetry in the flagpole and shadow case.

... [T] he shadow pattern cannot be instantiated in all the cases in which we provide origin-and-development explanations. Take any unilluminated object. It casts no shadow. Hence we cannot instantiate the shadow pattern to explain its dimensions. $^{37}$

However, unlike in the unificationist account, these kinds of considerations are now merely evidence that we have for or against nomological dependence. There is no need to compare the overall unificatory power of an "origin-and-development" pattern versus a "shadow" pattern.

A second example raised by Teller ${ }^{38}$ will clarify the difference. Bromberger ${ }^{39}$ also notes that the failure of symmetry has to do "... with the fact that whereas the period would not have been what it is if the length had not been what it is, the length would have been what it is whether the bob had been oscillating or not". Teller objects to Bromberger's way of capturing this intuition. Teller notes that it falters (in among other cases) when it comes to accounting for why the dispositional period of a pendulum does not explain the length of the pendulum (although it has this dispositional period whether it is swinging or not). Kitcher tackles this objection on behalf of unificationist accounts.

Someone may ...suggest that we can construct derivations of the dimensions of bodies from specifications of their dispositional periods, thereby generating an argument pattern which can be applied as generally as that instantiated in origin and

\footnotetext{
${ }^{37}$ Philip Kitcher. Explanatory Unification and the Causal Structure of the World. In P. Kitcher and W. Salmon, editors, Scientific Explanation, volume XIII of Minnesota Studies in the Philosophy of Science, pages 410-505. University of Minnesota Press, 1989, p 485.

${ }^{38}$ Paul Teller. On Why-Questions. Nô̂s, 8(4):371-380, 1974.

${ }^{39}$ Sylvain Bromberger. Why-Questions. In R. Colodny, editor, Mind and Cosmos, volume 3 of University of Pittsburg Series in the Philosophy of Science, pages 86-111. University of Pittsburg Press, 1966, 106.
} 
development explanations. This suggestion is mistaken. There are some objectssuch as the Earth and the Crab Nebula - which could not be pendulums, and for which the notion of a dispositional period makes no sense. Hence, the argument patterned proposed cannot entirely supplant our origin and development derivations, and, in consequence, acceptance of it would fail to achieve the best unification of our beliefs. ${ }^{40}$

On the view in this paper, the first question to ask is whether or not a simple pendulum law dealing with dispositional periods, $T$ (dispositional $) \approx 2 \pi \sqrt{\frac{L}{g}}$, would hold generally. As Kitcher argues, the answer to this question is clearly no. ${ }^{41}$ There are objects that do not have dispositional periods and yet have lengths (or more generally dimensions). Teller's question is whether we should take the dispositional periods of pendula to explain their lengths. Since the unificationist account works by demanding maximal unification, it is enough for a unificationist view to note that such a pattern would fail to be as generally applicable as other competitor patterns. ${ }^{42}$ This is not part of the view that I have put forward. On the view in this article we can still ask whether or not the dispositional period can explain the length for pendula (objects with dispositional periods). Although Kitcher is right that $T$ (dispositional) $\approx 2 \pi \sqrt{\frac{L}{g}}$ cannot apply generally, we can associate a condition of application with the dispositional version of the simple pendulum law. Namely, that it only applies to pendula (whether swinging or not). Once we have noted this restriction in application we need to ask if the length is sensitive to violations of this condition of applicability. That is, is the length of the object sensitive to whether or not the object under consideration is a pendulum? We have good reason to think that the answer is no. No restrictions are posed on the length of an object by it not falling under the class of pendula. In contrast, the length looks like it can still explain the dispositional period via the dispositional simple pendulum law. The dispositional period is sensitive to this condition of application. The dispositional period of an object is (by definition) sensitive to whether or not the object is a pendulum.

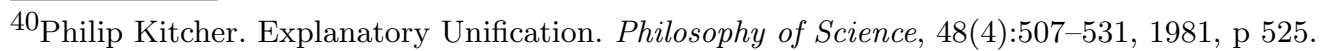

${ }^{41}$ The full view in Philip Kitcher. Explanatory Unification and the Causal Structure of the World. In P. Kitcher and W. Salmon, editors, Scientific Explanation, volume XIII of Minnesota Studies in the Philosophy of Science, pages 410-505. University of Minnesota Press, 1989, pp 484-87, also appeals to the idea of not allowing gerrymandered properties. The account in this paper, as well as causal accounts, also requires such a view.

${ }^{42}$ This "winner-takes-all" aspect of unficationist accounts has been criticised by James Woodward. Making Things Happen: A Theory of Causal Explanation. Oxford University Press, 2003, p 367.
} 
This brings me to a final problem that Teller raises when evaluating future promising avenues for understanding explanation.

Bromberger's account and for that matter Hempel's account of explanations are already not purely formal; for they rely on the concept of lawlikeness which is now widely recognised not to be a purely formal notion. It is natural to suspect that there are further non-formal aspects of contexts of discourse and explanation which are relevant to explanation and the relation of certain questions to their answers. By way of illustration, consider again the counterexample ... involving a pole. It is possible to describe sufficiently bizarre contexts in which ... a pole's dispositions for relations of physical geometry might be taken to explain its height. ${ }^{43}$

Van Fraassen has a particularly forceful example of the seeming context dependence of the directionality of explanation.

4.1. van Fraassen's tower. While the discussion in section 4 shows that the account is able to capture the asymmetry of some specific and traditionally troublesome cases, it does not rely on a relationship built to be asymmetric to do so. This means that it is not ruled out that there can be both cases where $a$ explains $b$ and ones where $b$ explains $a$. I think that this is a virtue of the account. For example, the nature of this solution provides a ready response to van Fraassen's case where a tower is constructed specifically to cast a shadow of a certain length at a certain time of the year. ${ }^{44}$ Some, including van Fraassen himself, have taken cases like this to cast doubts on the possibility of giving a both adequate and illuminating analysis of explanation in terms of necessary and sufficient conditions. Such cases seem to show that even the asymmetry of explanation can shift with context (since now the length of the shadow can explain the height of the tower after all). The solution to the non-symmetry problem given in this paper does not demand denying that there could be any explanation of the height of the tower from the length of the shadow. Rather, this account only denies that the application of the standard trigonometric relation supports such a law-based explanation. Even in van Fraassen's case this seems right. The case that van Fraassen puts forward is motivated by causal reasoning (although of a complicated kind going through the

\footnotetext{
${ }^{43}$ Paul Teller. On Why-Questions. Noûs, 8(4):371-380, 1974, pp 378-379.

${ }^{44}$ Bas van Fraassen. The Scientific Image. Clarendon Press, Oxford, 1980, pp 132-134.
} 
intentions of those ordering the construction of the tower, etc.). The solution here has not ruled out an explanatory role for such causal relations.

\subsection{Time-symmetric formulations of the laws versus time-symmetric explanations.}

Since the account of law-based explanation that I have suggested does not build asymmetry in by default, it leaves it open whether time-symmetric formulations of laws within our physical theories mandate time-symmetric explanations. ${ }^{45}$

It is often claimed that while predictions can sometimes also be explanations, retrodictions are not explanatory. ${ }^{46}$ On a nomological dependence account of explanation there is no simple answer to give as to whether retrodictions can be explanatory. Instead the question is bound up with how we regard the relationship between the formulations of our laws and time. Let me briefly show how we are left with two options.

The question that we have to answer is whether or not time-symmetric formulations of, say, laws of fundamental physics give us reason to think that there are laws of nature that guarantee the dependence of past states on future ones (as well the dependence of future states on past states). However, as we have seen again and again in this paper, the mere time-symmetric formulation of the laws does not guarantee symmetry when it comes to explanation. After all, we first have to decide what the associated conditions of application are. Among the questions that we would have to settle in order to decide this is whether we take the laws in question to apply to the nature of time or whether the existence of some particular kind of temporal order is rather a condition of application of the laws. To illustrate the difference in a dramatic way we can ask whether we take the laws in question to rule out the possibility of an apocalypse (in the sense of the end of time) taking place between the past state and the occurrence of some particular future one mandated by the laws. If we answer yes to that question, we open up for a symmetry of explanation from the temporal symmetry of the formulation of the laws. However, if we answer no then we do not take the time-symmetric laws to apply unrestrictedly and to be able to guarantee the dependence of

\footnotetext{
${ }^{45} \mathrm{I}$ will not discuss whether we should regard specific formulations as time-symmetric since this merits a longer discussion than this paper allows.

${ }^{46}$ See for example Barry Loewer. Counterfactuals and the second law. In H. Price and R. Corry, editors, Causation, Physics, and the Constitution of Reality: Russell's Republic Revisited, pages 293-326. Oxford University Press, 2007, p 294.
} 
past states on future ones (since they fail to guarantee that the past ones would not have existed had the future ones failed to). ${ }^{47}$

The upshot of this way of thinking of explanatory non-symmetry in general is that even if we acknowledge the existence of time-symmetric formulations of laws, it does not immediately follow that we will also have time-symmetric explanations. This is so even though it can give us reasons to accept such explanations. We have already seen how we typically have to be careful in noting any implicit restrictions in application of the laws before trying to read off relations of dependence from those laws. Figuring out exactly what these restrictions are is by no means a trivial matter, and it will often involve a rather sophisticated understanding of the whole theory of which the law is a part. This is, I think, as it should be. As the problem of non-symmetry shows, we cannot understand what depends on what simply by being told that there is a lawlike connection between two phenomena.

\section{Conclusion}

The account of law-based explanation that I have proposed does not fit neatly as a development of any of the traditional accounts. It is broader than the causal account since not only causal relations serve as the basis for explanation and for introducing explanatory non-symmetries. It is narrower than the deductive-nomological account: it is not sufficient for explanation that the phenomenon to be explained is subsumed under some law.

The account proposed is also different from interventionist accounts, even though it shares the importance placed on considerations of counterfactual scenarios. Sophisticated interventionist accounts, such as Woodward's, still employ a solution to the non-symmetry problem that relies crucially on causal relations. ${ }^{48}$ However, the motivating intuition of thinking of explanation in terms of dependence is close to Woodward's account. It also shares with Woodward's account an acknowledgement that laws do not have to apply unrestrictedly in order to do explanatory work. However, the account that I have outlined does not rely on causal notions to account for these features.

\footnotetext{
${ }^{47}$ For an argument along similar lines, but from a very different starting point see Tim Maudlin. The Metaphysics Within Physics. Oxford University Press, 2007, Chapter 4: On the passing of time.

${ }^{48}$ Although the solution is given in terms of interventions, these are cashed out in causal terms in James Woodward. Making Things Happen: A Theory of Causal Explanation. Oxford University Press, 2003, section 3.1.3.
} 
Finally, although I have defended the view that we can address the problem of explanatory nonsymmetry within a law-based account of explanation, I have not rescued the deductive-nomological account. How far are the suggestions that I have made here friendly to the sensibilities behind the deductive-nomological account? The answer is, I think, surprisingly largely so. The account that I have presented here is metaphysically neutral about the nature of laws and compatible both with non-Humean primitivism about the laws and Humean reductionism. For someone with Hempel's ${ }^{49}$ commitments, projects that make use of counterfactuals, such as mine, can be taken to "...shed light on the concept" although they cannot provide satisfactory explications. This is a feature that my account shares with causal accounts. How large this cost is depends on how optimistic we are about there being a successful explication that respects Hempelian strictures.

Of course, the account in this paper is far from a full account of scientific explanation. I have not addressed probabilistic and statistical explanations at all, nor have I given a comprehensive account of explanatory relevance, approximations, idealisations, and modelling practices. Nonetheless, I hope to have shown that the elegance of a causal solution to the problem of explanatory asymmetries should not lead us to base our investigations of these aspects of explanation on causal foundations alone.

Taking law-based explanations to be able to provide information about relations of nomological dependence without relying on causal underpinnings has consequences for debates in philosophy beyond developing a model of explanation. It affects our understanding of cases where we may be more confident about having an explanation than we are about having a causal relation: for example, explanations of the spin of one particle by the spin of the other in certain quantum mechanical (EPR style) situations. It also has consequences for arguments that use explanatory connections to argue for the existence of causal ones. ${ }^{50}$ Lastly, it gives us the start for a framework for understanding certain disputes about explanatoriness. In addition to denying the truth or the status as a law of the putative law, one could now also challenge the claim that the law supports the dependence in question. ${ }^{51}$ To settle the question we have to take a stance on what the appropriate

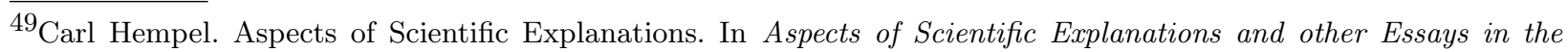
Philosophy of Science, pages 331-496. The Free Press, New York, 1965, p 339.

${ }^{50}$ As we see for example in Harvey Brown. Physical Relativity: Space-time structure from a dynamical perspective. Oxford University Press, 2005.

${ }^{51}$ For a clear example of such a strategy see Sheldon Smith. Symmetries and the explanation of conservation laws in the light of the inverse problem in lagrangian mechanics. Studies in History and Philosophy of Modern Physics, 39 (2):325-345, 2008.
} 
conditions of application of the law are, and, on the view in this article, the debate over this is not explanatorily innocuous. 